\title{
TEORÍAs DEL DESARROLlO CAPITALISTA. UNA EVALUACIÓN COMPARADA ${ }^{1}$
}

\author{
Ignacio Trucco*
}

Fecha de recepción: 12 de noviembre de 2011. Fecha de aceptación: 25 de enero de 2012.

\begin{abstract}
RESUMEN
Este trabajo tiene por objetivo comparar un conjunto de teorías del desarrollo capitalista indagando las suposiciones que éstas construyen sobre la naturaleza y la dinámica de la sociedad. El trabajo busca mostrar posibles paradojas al momento de intentar interpretar el cambio social suponiendo sociedades estáticas. Para ello, primero se oponen las ideas de la teoría económica convencional con el tratamiento de la teoría del crecimiento desde el modelo Harrod-Domar y la interpretación de la dinámica histórica de Rostow recuperando la crítica de Germani y Medina Echavarría. En una segunda instancia se desarrollan los aportes de Hirschman-Myrdal y de Prebisch-Singer, concluyéndose con la crítica dialéctica desarrollada por Furtado. Finalmente se indagan estos aspectos en el giro neoclásico de la teoría del desarrollo basado en los presupuestos de la Nueva Economía Institucional.
\end{abstract}

Palabras clave: desarrollo, capitalismo, teorías comparadas, dialéctica, paradojas.

\section{Theories of Capitalist Development a Comparative Evaluation}

\begin{abstract}
This work seeks to compare a set of theories on capitalist development by investigating the assumptions made on the nature and dynamics of society. This work aims to show possible paradoxes when interpreting social change under the assumption of static societies. First, ideas from conventional economic theory are contrasted with the theoretical treatment of growth in the Harrod-Domar model and Rostow's interpretation of historical dynamics, taking into account critiques from Germani and Medina Echavarría. Secondly, Hirschman-Myrdal's and Prebisch-Singer's contributions are expanded upon, concluding with a critique developed by Furtado. Finally, the work investigates these aspects on the neoclassical side of development theory based on proposals from New Institutional Economics.
\end{abstract}

Key Words: development, capitalism, comparative theory, debate, paradoxes.

1 Este trabajo forma parte de la tesis doctoral del autor.

* Docente de la Facultad de Ciencias Económicas de la Universidad Nacional del Litoral, Santa Fe, Argentina. Correo electrónico: ignacio.trucco@gmail.com 


\section{DES THÉORIES DU DÉVELOPPEMENT CAPITALISTE. UNE ÉVOLUTION COMPARÉE Résumé}

Ce travail a pour but de comparer un ensemble de théories du développement capitaliste en enquêtant sur les suppositions qu'elles élaborent quant à la nature et la dynamique de la société. Le travail vise à montrer de possibles paradoxes surgissant quand on tente d'interpréter le changement social en supposant des sociétés statiques. Pour cela, en premier lieu, les idées de la théorie économique conventionnelle sont opposées au traitement de la théorie de la croissance à partir du modèle Harrod-Domar et l'interprétation de la dynamique historique de Rostow en récupérant la critique de Germani et Medina Echavarria. En second lieu, sont développés les apports de Hirschman-Myrdal et de Prebisch-Singer, concluant par la critique dialectique développée par Furtardo. Finalement, ces aspects sont recherchés dans la forme néoclassique de la théorie du développement basée sur les présupposés de la Nouvelle Économie Institutionnelle.

Mots clés: développement, capitalisme, théories comparées, dialectique, paradoxes.

\section{TEORIAS DO DESENVOLVIMENTO CAPITALISTA. UMA AVALIAÇÁO COMPARADA. Resumo}

Este trabalho tem como objetivo comparar um conjunto de teorias do desenvolvimento capitalista indagando as suposiçóes que estas constróem sobre a natureza e a dinâmica da sociedade. O trabalho busca mostrar possíveis paradoxos ao momento de tentar interpretar a mudança social supondo sociedades estáticas. Para tanto, primeiro se opóem as idéias da teoria econômica do crescimento desde o modelo Harrod-Domar e a interpretação da dinâmica histórica de Rostow recuperando a crítica de Germani e Medina Echavarría. Num segundo momento, se desenvolvem as contribuiçóes de Hirschman-Myrdal e de Prebisch-Singer, concluindo com a crítica dialética desenvolvida por Furtado. Finalmente, se indagam estes aspectos no giro neoclássico da teoria do desenvolvimento baseada nos pressupostos da Nova Economia Institucional.

Palavras-chave: desenvolvimento, capitalismo, teorias comparadas, dialética, paradoxos.

\section{资本主义发展理论中的流动和社会：比较评估}

摘要

本文试图通过研究关于社会特性和动态的假设, 比较资本主义发展理论。研 究目标是求证静态社会假设条件下对社会变化的解释可能存在矛盾之处。 首先, 考虑到赫尔马尼和玛蒂娜・埃恰瓦利亚的批判, 本文对传统经济理论 思想与哈罗德多马增长模型和罗斯托对经济增长历史动态的解释进行了比 较。其次, 本文拓展了赫希曼-缪尔达尔和普雷维什-辛格的理论贡献, 并以 富尔塔多的批判性理论作为结尾。最后, 本文还研究了以制度经济学为基础 的新古典流派的发展理论。

关键词: 发展资本主义比较理论争论 悖论 


\section{EL INCÓMODO LUGAR DEL DESARROLLO: ENTRE LA UTOPÍA Y LA DINÁMICA}

En el campo de las ciencias de la sociedad hay siempre un capítulo reservado a la consideración de las relaciones que tejen enunciados con fines normativos y enunciados con pretensiones de objetividad. En este lugar del campo se ha abierto un abismo cuya solución se ha manifestado predominantemente parcial e inconclusa.

La Crítica de Kant ha tenido una influencia decisiva en cientistas como Weber o Simmel (para el caso de Weber ver González García, 1988) donde el modelo de funcionamiento heredado promovió una radical separación entre filosofía teórica y práctica si bien, como puede leerse en Weber, las valoraciones prácticas tienen siempre un papel preponderante en la significación de la actividad intelectiva; sobre esto Weber sintetiza:

Hay y sigue habiendo -esto es de lo que para nosotros se trata- para cualquier época una infranqueable diferencia entre si una argumentación se dirige a nuestra sensibilidad y a nuestra capacidad de entusiasmarnos por fines concretos y prácticos o por formas y contenidos culturales, o de si se dirige, en cuanto esté en discusión la validez de normas éticas, a nuestra conciencia o, finalmente, a nuestra facultad y necesidad de ordenar intelectualmente la realidad empírica de modo que reclame su validez como verdad empírica. Y esta proposición permanece siendo exacta, a pesar de que, como ya se indicó, aquellos supremos 'valores' del interés práctico son y continuarán siendo siempre de decisiva importancia para la orientación que la actividad ordenadora del pensamiento introduce continuamente en el dominio de las ciencias de la cultura (Weber, 1956: 431-432).

La relación entre dos campos que se suponen plenamente diferenciados está siempre referenciada en la historia del pensamiento económico: la economía normativa y la positiva tejen esta historia. Ricardo Gómez, por ejemplo, polemiza sobre este punto clave de la teoría económica dominante, el que es presentado como una pretensión de neutralidad, a lo cual el autor muestra cómo ésta es una contradicción en sí (Gomez, 2002).

Las teorías del desarrollo están particularmente ubicadas en este incómodo lugar donde lo normativo y lo positivo traspasan sus límites respectivos, muchas veces sin una explicitación clara. Este trabajo trata de aportar al esclarecimiento de este punto, sobre todo a partir de un intento de mostrar de forma metódica que, a diferencia de lo que se sostiene de manera extendida en el campo de las teorías del desarrollo, los problemas que éstas manifiestan tienen en esencia un fundamento positivo y no normativo. Se busca por lo tanto esclarecer las características de este "incómodo" lugar mostrando qué principio positivo 
es el que permanece no explicitado. A partir de lo desarrollado es posible ver cómo esta dificultad está íntimamente vinculada al tratamiento del movimiento espacio-temporal de la sociedad en cada perspectiva el cual, además, se funde con las suposiciones que estos autores realizan sobre la naturaleza del hombre y su socialización. Por lo tanto, la lectura que se propone de los distintos enfoques se estructura a partir del siguiente principio: cada crítica recae sobre la forma en que el movimiento es tratado; al mismo tiempo, cada una presenta límites inherentes en la imposibilidad de integrar el movimiento en una concepción de la naturaleza del hombre y su socialización eminentemente estática.

Para comenzar a despejar los límites de lo positivo y lo normativo en las teorías del desarrollo podemos recuperar el enfoque weberiano arriba reseñado.

En primer lugar, existe un conjunto de afirmaciones que, se supone, componen una teoría positiva donde se explicitan las relaciones que determinan sincrónica y diacrónicamente la vida de las sociedades.

En segundo lugar, existe una meta deseable, de carácter normativo y más o menos general, no fundada científicamente.

En tercer lugar, de los momentos anteriores se derivan dos posibilidades: primera, los sistemas sociales tienden, por razones positivamente fundadas, a una meta que coincide con la meta normativa. Segunda, los sistemas sociales no necesariamente tienden a la meta normativa pero es posible conocer los factores que pueden determinar, por razones positivamente fundadas, un cambio de trayectoria.

Es ilustrativo cómo Aldo Ferrer, en su popular historia económica argentina, sintetiza esta tensión marcada en estos puntos. Ferrer afirma que

[...] no ha cambiado la naturaleza del proceso de desarrollo económico. [...] El desarrollo económico sigue siendo un proceso de transformación de la economía y la sociedad fundado en la acumulación de capital, conocimientos, tecnología, capacidad de gestión y organización de recursos, educación y capacidades de la fuerza de trabajo, y de estabilidad y permeabilidad de las instituciones, dentro de las cuales la sociedad transa sus conflictos y moviliza su potencial de recursos. [...]

El proceso resulta indelegable en factores exógenos los cuales, librados a su propia dinámica, sólo pueden desarticular un espacio nacional [...] y, por lo tanto, frustrar los procesos de acumulación, es decir, el desarrollo (Ferrer, 2008: 462-463).

Esta cita condensa los momentos que se identifican arriba y la pregunta que puede hacerse es si Ferrer distingue aquellas ideas que responden a su jerarquía moral de aquellas ideas que refieren a la naturaleza y dinámica de la sociedad.

Müller (1996: 16-18) destaca la presencia (particularmente intensa en los últimos años) de enunciados normativos en las teorías del desarrollo y aclara que en 
ellas la búsqueda de una meta político-moral es también su explicitación, con lo cual su debilidad puede convertirse en una fortaleza.

La explicitación o el ocultamiento de la meta normativa suele tomar una posición predominante en debates sobre el desarrollo y lo que puede inducir una invisibilización de un debate necesario sobre las características de las teorías de la sociedad que cada enfoque construye.

Ciertas teorías suelen ser denunciadas por no explicitar la meta normativa a la cual suponen evidente. Esta crítica suele recaer sobre los modelos neoclásicos y keynesianos de crecimiento $\mathrm{y}$, en términos generales, oficia de punto de partida de la crítica teórica. En este trabajo se cree que este tipo de lecturas pueden invertirse: los modelos ocultan, sobre todo, la suposición de argumentos referidos a la naturaleza de la sociedad y su funcionamiento. Estas suposiciones no explicitadas toman relevancia cuando en razón de heterogeneidades espaciotemporales se produce un distanciamiento entre la teoría y los fenómenos estudiados.

En segundo lugar y desprendiéndose de lo anterior, cuando enunciados positivos quedan ocultos en la cadena de argumentos, suele ocultarse a la vez una explicación tautológica del cambio social. En realidad esto ocurre, como se verá, porque una teoría de la dinámica social que supone una sociedad cualitativamente estática no puede dar cuenta del cambio histórico y concretamente no logra dar cuenta de la formación de la modernidad capitalista. En este contexto surgen falsas explicaciones que se replican recurrentemente en la teoría del desarrollo. ${ }^{2}$ En buena medida la teoría del desarrollo nace denunciando esta incapacidad pero no logra superar esta paradoja.

\section{EL DEBATE SOBRE EL DESARROLLO EN EL SIGLO XX: ENTRE LA UTOPÍA BIENESTARISTA Y LA DEPENDENCIA}

\section{Entre la modelistica del crecimiento y la dualidad sociedad tradicional/moderna}

Tras la finalización de la Segunda Guerra Mundial, la organización del mundo capitalista proyectó nuevos imaginarios nacidos al amparo de los Estados de bienestar-keynesianos, la producción fordista, el consumo masificado y la

2 En un contexto muy diferente pero con una estructura similar Moncayo Jiménez afirma que "el argumento de tales enfoques teóricos era un tanto tautológico: la aglomeración de productores en una localización proporciona ventajas y estas ventajas, precisamente, explican la aglomeración. Estas teorías suponen lo que están tratando de entender" (2001: 14). 
estructuración corporativa de ciertos principios básicos de socialización con la particular centralidad de la relación salarial (Jessop, 2008: 67-74). En este contexto socio-histórico, la teoría económica desarrollada en los países centrales trató de racionalizar estos procesos de modo tal de exponer principios universales lógicamente articulados desprovistos de toda particularidad histórico concreta. El modelo de referencia que tuvo una incidencia particular fue aquél desarrollado por Harrod y Domar en el cual, tras definirse los determinantes del crecimiento del producto por persona, se intenta encontrar aquella tasa de ahorro que estabiliza el modelo en una senda de crecimiento en la cual lo previsto coincide con lo realizado y el producto efectivo coincide con el potencial. ${ }^{3} \mathrm{Si}$ bien esto redunda en un modelo esencialmente inestable, permite fijar el criterio de lectura de una sociedad auto-regulada que se "desarrolla" sobre la base de la acumulación de capital físico una vez especificadas las determinaciones tecnológicas que convierten el incremento de capital físico en incremento del producto.

Pero la hipótesis clave del modelo (y probablemente la más fuerte de todas sus hipótesis) no está en los determinantes del crecimiento del producto por habitante sino, sobre todo, en el tipo de sociedad supuesta compatible con dicha dinámica social: una sociedad organizada sobre la base de decisiones descentralizadas de individuos que determinan consumo, ahorro e inversión bajo una racionalidad definida por la acción con arreglo a fines en los que no hay, necesariamente, otro principio que ordene la conducta en el proceso de acumulación de capital.

Estas determinaciones permiten pensar un sistema de decisiones individuales auto-regulado cuyos comportamientos agregados, no conscientes ni deliberados, comportan ciertas regularidades que pueden presentarse como leyes de movimiento.

Esta suposición no explícita es la que comienza a generar resistencias entre los teóricos que observan sociedades periféricas que no responden con facilidad a estas exigencias. Hans Singer denuncia explícitamente esta pretensión como una operación al menos dudosa: "Ésta era una aplicación directa de las ideas desarrolladas en los países industriales, por los países industriales, para los países industriales, y basadas en conceptos aplicables a los países industriales" (Singer, 1981 a: 48-49).

3 Para ver un desarrollo del modelo en la versión de Harrod y en la de Domar por separado recurrir a Galindo y Malgesini (1994: 11-27). 
En cierto sentido, el problema del desarrollo capitalista en el modelo de Harrod-Domar ya está resuelto antes de comenzar a ser estudiado. Singer pone de manifiesto esta inversión que el enfoque supone y presta atención a la exogeneidad de la tasa de crecimiento de la población: suponer esta exogeneidad es poner por fuera lo que debería nacer dentro del sistema de argumentación. En este sentido Singer afirma que

el problema real del desarrollo a largo plazo, sin duda, consiste en equiparar a la generación actual de niños, alimentarlos, vestirlos, albergarlos, educarlos y adiestrarlos en forma tal que se vuelvan productores más eficientes que sus antecesores. Ése es el proceso del desarrollo económico en un sentido mucho más importante que el proceso de la formación de capital físico (Singer, 1981a: 53-54).

No es precisamente un problema normativo el que se destaca aquí. Por el contrario, los modelos de crecimiento realizan una apuesta ontológica no explicitada sobre el tipo de sociedad existente, donde se supone una naturaleza específica del hombre y (si no se resuelve, al menos sí) se acota el problema moral humano en la producción y reproducción de su vida material. Por lo tanto, la acumulación capitalista con todas sus determinaciones sobre el hombre y la sociedad, resulta para el enfoque de la teoría ortodoxa una suposición.

Una figura que aparece en un lugar controversial, ambiguo y sobre todo contradictorio, es la de Walt Whitman Rostow quien suele representar de manera simultánea una defensa de la jerarquización del cambio técnico en la interpretación de la dinámica social y a la vez inicia un debate complejo y más amplio sobre la naturaleza del desarrollo capitalista.

Este autor introduce la noción de crecimiento del producto y de control racional de la técnica como su fundamento, estructurando una dinámica histórica. En ella las sociedades atraviesan etapas sucesivas y despliegan allí formas de organización social que ponen en especifica relación al hombre con el conocimiento cientifico tecnológico de modo tal que finalmente los modelos de crecimiento keynesianos toman pleno sentido.

En algunos casos, las ideas de Rostow se han interpretado como la afirmación de sólo una trayectoria posible frente a la cual todas las sociedades del mundo enfrentan su contraste; Hirschman, en este sentido, entiende que la de Rostow es una "posición monoeconómica" (Hirschman, 1984: 23).

Pero más allá de la apreciación de Hirschman, Rostow se ve obligado a trascender los límites del lenguaje formal a fin de poder interpretar el cambio histórico. Con lo cual debe atravesar un momento clave: el proceso de transformación de un tipo de sociedad a otra, y por lo tanto la necesidad de definir 
si existen sociedades cualitativamente diferentes o si por el contrario sólo existen distancias cuantitativas. Esto en la literatura de América Latina será recibido como el dualismo de lo "tradicional" versus lo "moderno".

Resulta importante indagar en Las etapas del crecimiento económico dos elementos: cuál es el hilo conductor del movimiento y qué tipo de objeto se supone tal que admite dicho movimiento. En cuanto al primer punto, hay consenso general en destacar la importancia que Rostow daba al progresivo control de la ciencia y la técnica modernas y el incremento de la productividad general de los factores (Gutiérrez, 2004: 298). Por lo tanto puede pensarse la lógica del movimiento basada en un incremento del control técnico-científico de la naturaleza conducido a la producción de bienes de capital (privado y social) y bienes de consumo (básico y duradero). Rostow afirma:

Pero el hecho fundamental relacionado con la sociedad tradicional era que existía un tope al nivel de la producción obtenible per cápita. Este límite provenía del hecho de que no eran asequibles las posibilidades cientificas y técnicas modernas o que no se podian aplicar en forma regular y sistemática [...] el nivel de la productividad estaba limitado por lo inaccesible de la ciencia moderna, de sus aplicaciones y del marco intelectual (Rostow, 1961: 16-17). -Las cursivas son nuestras-.

Pero una afirmación de este tipo tiene las mismas implicaciones tratadas en el caso del modelo Harrod-Domar, pero a diferencia de éstos, Rostow no puede silenciarlo por completo pues, por el contrario, si pretende hacer una historia debe explicitar qué hay de nuevo, qué distingue a la sociedad tradicional de la moderna, qué cambia y qué permanece. Pero ello, a su vez, impacta sobre la construcción del objeto que adopta este movimiento: el hombre y la sociedad.

Rostow, a diferencia de Marx (i!), no elabora de forma explícita una teoría del hombre y de las relaciones que instituyen a la moderna sociedad capitalista. Por el contrario, éste construye sus premisas desde un enfoque ante todo empirista, y procede desde la identificación de ciertas regularidades hacia la enunciación de principios generales y abstractos.

Para el autor, entre la sociedad tradicional y la moderna, media un proceso de secularización en la relación que el hombre teje con la naturaleza y puesto allí el acento, supone que aquél queda constituido en sí mismo y en sus representaciones frente a la naturaleza física que lo circunda.

Al respecto Rostow afirma: "el hombre no necesita considerar a su medio ambiente material como un factor obsequiado por la naturaleza y la Providencia, sino como un mundo ordenado que, si se le comprende en forma racional, puede ser manejado de modo que rinda un cambio productivo" (Rostow, 1961:32). 
Por lo tanto Rostow parte de una doble ontología: por un lado la existencia de un ente individual que asume una relación específica con la técnica y la realidad material y, por otro lado, la existencia de una instancia institucional, a la manera de un conjunto de reglas que son funcionalmente compatibles.

Las dificultades aparecen en las indeterminaciones entre uno y otro, en las arbitrariedades y en las indefiniciones que caracterizan finalmente a la obra. El determinismo de la técnica será su refugio.

Furtado es categórico al respecto:

Su teoría resulta, sin embargo, demasiado simplista, limitándose a recalcar la existencia de un camino óptimo para el desarrollo de la producción, determinado por el progreso tecnológico y por las elasticidades de la demanda [...] Después de todo, la teoría de la producción rostowiana no explica el tránsito de las formas de producción tradicionales hacia las formas industriales (Furtado, 1974: 132).

Los aportes de Rostow generaron reacciones de diverso tipo. Los teóricos latinoamericanos de la modernización respondieron de forma directa aun sosteniendo como hipótesis de interpretación histórica el pasaje de sociedades tradicionales a sociedades modernas. Teóricos de la talla de Gino Germani y José Medina Echavarría iniciaron una crítica en cierto sentido fundacional de la sociología en América Latina. Estos autores comprendieron largamente las dificultades y limitaciones del enfoque de Rostow, si bien entendían que el proceso de formación de sociedades modernas era el objeto clave a ser caracterizado.

Marcos Roitman resume parte de las reflexiones de Medina Echavarría y Germani y destaca cómo las transformaciones políticas, culturales e institucionales desempeñan un papel clave en el proceso de modernización en los países subdesarrollados. En este sentido Roitman destaca que "Si la racionalidad-dirá Medina Echavarría- se manifiesta en un proceso general de desarrollo y progreso científico y técnico, será en último término el contenido democrático de la voluntad y decisión política, la clave para legitimar el proceso de cambios sociales" (Roitman, 2005: 40).

Por otra parte, para el caso de Germani afirma que "Germani sintetiza recalcando que toda modernización conlleva un proceso de cambio en las estructuras sociales y de poder cuya dinámica desemboca en un proceso de secularización" (Roitman, 2005: 43).

En definitiva, estos autores ponen de manifiesto cómo el problema de la modernización capitalista no está realmente resuelto en Rostow, quien deja sin explicación el componente fundamental: el cambio societal como una totalidad cultural, institucional y material. La profundidad y el desarrollo del análisis de 
Germani y Medina Echavarría no obstan a que exista cierta afinidad con el método empirista y lógico-funcionalista y con una presencia recurrente de afirmaciones teleológicas.

\section{La respuesta frente al subdesarrollo: de la industrialización a la dialéctica de la dependencia}

La idea de que las sociedades atraviesan etapas en un proceso de secularización en el tratamiento de la técnica moderna y allí despiertan un proceso de acumulación auto-centrado tal que convergen, fue intensamente cuestionada durante las décadas de los sesenta y los setenta del siglo pasado.

Albert Hirschman y Gunnar Myrdal fueron autores paradigmáticos de esta crítica y generaron una lectura de la dinámica social en la cual preveían el incremento de las asimetrías entre países. El optimismo que conllevaba la obra de Rostow aquí era puesto en duda.

Hirschman sintetiza estos aportes,

Gunnar Myrdal invocó el principio de la causación acumulativa para tratar de entender la razón de las persistentes y crecientes disparidades del ingreso dentro de los países; pero la noción se extendió sin dificultad a los contactos existentes entre países [...] Independientemente de Myrdal, yo había elaborado ideas similares: el "efecto de estela" de Myrdal -los factores que propician el aumento de la disparidad- se convirtió en mi "efecto de polarización", mientras que su "efecto de dispersión" -los factores que propiciaban la difusión de la prosperidad de las regiones ricas a las pobres- fue mi "efecto de filtración" (Hirschman, 1984: 30).

La idea de que existe un conjunto de fuerzas que determinan la trayectoria de las sociedades y que esas fuerzas, puestas en movimiento, tienen una dinámica inherente tal que las mismas se refuerzan y los resultados se polarizan en el espacio, constituye el locus interpretativo del esquema Myrdal-Hirschman.

Los modelos se presentan como una descripción de dinámicas espaciales integradas capaces de captar diferencias entre espacios, pero el análisis no renuncia a las formas abstractas de los modelos tradicionales. Por el contrario, MyrdalHirschman intentan mostrar situaciones de desequilibrio y la posibilidad de incorporar dimensiones cualitativas en el análisis, pero sosteniendo implícitamente una importancia sustantiva de la forma general del modelo sobre el contenido específico que éste adquiera.

Myrdal expresa sintéticamente esta forma general de la causación circular acumulativa: 
Si cualquiera de los dos factores cambiase, se produciría también inevitablemente un cambio en el otro factor, lo que iniciaría un proceso acumulativo de interacción mutua en el cual el cambio experimentado por un factor estaría apoyado de manera continua por la reacción del otro factor, y así sucesivamente en forma circular (Myrdal, 1959: 28).

La forma general del argumento acabará teniendo una particular influencia en la llamada "economía de la localización industrial”. La Nueva Geografía Económica estudiará modelos dinámicos de localización en los cuales tomará esta idea de causas acumulativas y circulares que definen estructuras espaciales caracterizadas por asimetrías crecientes (ver Fujita, Krugman y Venables, 1999). Estos modelos pusieron el acento en la forma y sus explicaciones circulares han tendido a ser casi tautológicas (Moncayo Jiménez, 2001: 14). Los argumentos se han expuesto de la siguiente forma: dadas las diferencias es posible que éstas no se corrijan sino, por el contrario, se amplíen. Puede verse cómo tal conclusión está implícita en las premisas del modelo siendo ésta, sobre todo, una descripción de su dinámica.

Los modelos de la Nueva Geografía Económica llegaron sistemáticamente a resultados caracterizados por múltiples equilibrios en las estructuras geográficas, con lo cual la localización específica acaba siendo un "accidente de la historia" y en cierto punto azaroso (Moncayo Jiménez, 2001), siendo ésta una debilidad significativa que el mismo Krugman critica a sus antecesores como Von Thünen, Lösh y Christaller. En este sentido el mismo Krugman ejemplifica: "si Filadelfia, en vez de Nueva York, se hubiese establecido como centro de la industria financiera en 1869, este liderazgo sería igualmente sostenible hoy en día que el actualmente existente" (Krugman y Fukujita, 2004: 182). Este enfoque tiene límites de orden sustancial, dado que acaba reduciendo todo problema a las condiciones de origen.

Pero debe destacarse que estos enfoques tienen un impacto importante en el campo de la política pública, pues abren a las instancias de gobierno una ventana a la intervención con probabilidades de éxito. Esto, paradójicamente, proviene de la propia indeterminación de los modelos: si toda estructuración espacial es posible, la acción estatal tiene un margen de maniobra no desdeñable y está en condiciones de torcer la dinámica.

Hirschman afirma: "En todo caso, tanto Myrdal como yo consideramos los efectos de polarización como fuerzas que pueden ser combatidas y neutralizadas por las políticas públicas" (1984: 31).

Si bien estos teóricos han puesto el acento en la forma general, otros han tomado partido sobre un contenido específico cuyo impacto trascendió 
largamente los límites de la teoría económica. Dos autores son probablemente los más destacados en los orígenes de una discusión teórico-práctica que pensó el subdesarrollo a partir de la especificidad de la relación desarrollo-subdesarrollo.

Se hace referencia a la tesis Prebisch-Singer del deterioro de los términos de intercambio. La tesis de Singer del deterioro secular de los términos de intercambios encuentra sus razones en un reparto desigual de los beneficios de los incrementos de la productividad en los distintos sectores; así el autor expresaba: "el progreso técnico de las industrias manufactureras se reflejó en un aumento de los ingresos, mientras que el proceso técnico de la producción de alimentos y materias primas de los países subdesarrollados se reflejó en una disminución de los precios" (Singer, 1981b: 74).

Esta idea se apoya en un conjunto de justificaciones (se verá por qué se utiliza esta palabra) que suponen una determinada dinámica de la demanda y oferta de productos primarios y manufacturados tal que los distintos grupos sociales que participan de la producción de mercancías en los distintos países alcanzan un reparto del excedente determinado. Singer concluye que ese reparto desfavorece secularmente a los países productores de bienes primarios con lo cual no estarían nunca en condiciones de superar las características del subdesarrollo. Singer afirma: "Los países industrializados han tenido lo mejor de ambos mundos, como consumidores de bienes primarios y como productores de artículos manufacturados; los países subdesarrollados han tenido lo peor de ambos mundos, como consumidores de manufacturas y como productores de materias primas" (Singer, 1981b: 76).

Rolando Astarita ensaya una crítica a este enfoque en sintonía con lo que se ha desarrollando en este trabajo. Este autor pone de manifiesto los elementos no explicados de la teoría que son a la vez elementos fundamentales de esta interpretación. En este sentido Astarita afirma acerca de los problemas de la hipótesis del deterioro de los términos de intercambio, que:

En primer lugar, y tal vez el más serio, es que en su primer argumento Prebisch (y puede extenderse a Singer) termina recurriendo a una explicación basada en las relaciones de fuerza entre los sindicatos y el capital; y en las relaciones de fuerza de mercado entre los capitales de los países adelantados y los capitales de los países subdesarrollados. Es que Prebisch sostiene que los mark-up, o recargos, sobre los costos, son diferentes en los países adelantados y atrasados. Pero, ¿cuál es el nivel de ese recargo? ¿Cómo se establece? No hay teoría para esto, salvo decir que el nivel del mark-up depende del nivel de fuerza para establecer el mark-up. [...] Por eso este tipo de justificaciones, carentes de una perspectiva general, desembocan en el estudio de casos particulares (Astarita, 2010:139). -Las cursivas son nuestras-. 
Que estos enfoques desemboquen en el estudio de casos particulares puede traducirse como la incapacidad para dar cuenta de principios generales que conserven unidad y a la vez permitan penetrar el despliegue espacio-temporal del capitalismo. Estos enfoques resultan incapaces de poner en el centro de la escena lo constitutivo del sistema social y la formación histórica de la sociedad no es, en sí, un problema científico. Esta dificultad ontológica es el punto de partida de la crítica que realiza Rolando Astarita desde la tradición hegeliana y marxista.

Astarita trata esta temática y titula un capítulo de la obra citada como "Dependencia, cuestiones metodológicas a la luz de la tradición hegeliana y marxista" (2010: 65-85) y en él desarrolla la lectura de Palma (1987) y Blomström y Hettne (1990) sobre los errores que condujeron al derrotero de la "corriente de la dependencia" (CD) y el tipo de salida que éstos proponen basada en la "interacción dialéctica". En general Astarita se encuentra en sintonía con estos autores frente al diagnóstico que realizan sobre la CD; sin embargo muestra los límites de la "interacción dialéctica" que éstos desarrollan. Para ello afirma que

Esta perspectiva es superior al enfoque mecánico y rígido de las oposiciones abstractas y, como dice Hegel, nos pone 'en el umbral' del concepto dialéctico, pero no garantiza un tratamiento superador de las antinomias y, por eso mismo, en tanto se insista en permanecer en este plano, deviene estéril. En otras palabras, no brinda una salida porque nunca se puede precisar la manera en que actúa la mentada interacción. Por esta razón el 'tercer enfoque' de la dependencia, a pesar de apuntar en la dirección correcta, no pudo avanzar mucho más allá de plantear la necesidad de tener en cuenta la interacción entre 'el general' y 'los particulares' (o los 'singulares') (Astarita, 2010: 74).

Más adelante Astarita muestra cómo estos límites están presentes en la interpretación sociológica del subdesarrollo de Cardoso y Faletto (2007) a la cual Palma (1987) entendía como una interpretación "superadora".

En este sentido, Astarita advierte sobre los alcances de teorías estáticas de la sociedad frente al problema del despliegue histórico de la "moderna sociedad burguesa” (según la afamada expresión que Marx acuña en el Manifiesto).

Distintos autores de las teorías de la dependencia realizaron aproximaciones a la interpretación del subdesarrollo en el contexto de una teoría más general de la historia de la moderna sociedad burguesa. No sólo Cardoso y Faletto pueden ser citados en este sentido; también Celso Furtado ha reflexionado sobre el devenir histórico de la acumulación capitalista, destacando las tradiciones ilustradas del progreso social modernista. 
A partir de una lectura, quizá algo esquemática, de la filosofía de la historia de Hegel, Furtado reconoce cuál es el desafío que no lograron alcanzar los economistas tradicionales,

"El avance de las técnicas tendió a ser visto como un medio de superar la escasez de un factor de producción, a nivel de una unidad productiva. Esa visión [...] es el origen de muchas de las dificultades con que tropezarán los economistas para adoptar un enfoque dinámico de los procesos económicos y percibir en ellos algo más que una simple secuencia de situaciones estáticas" (Furtado, 1983: 17). -Las cursivas son nuestras-.

Es importante destacar esta idea: la necesidad de superar la visión fragmentaria de la realidad social y comprender que para penetrar su transformación espacio temporal se requiere un principio que otorgue inteligibilidad al movimiento donde cada momento defina su existencia en una relación con dicho principio y por lo tanto con el resto de los momentos que hacen a la historia como totalidad.

En este sentido, Furtado interpela el objeto de la teoría clásica del desarrollo que ha sido interpretado como exógeno al hombre, objetivado en sus propios términos, o aun como causa explicativa de transformación de la sociedad, siendo así el motor inmóvil de la historia. A ello Furtado opone una visión diferente:

Progreso técnico es en realidad una expresión vaga que en su uso corriente cubre el conjunto de las transformaciones sociales que hacen posible la persistencia del proceso de acumulación y por consiguiente la reproducción de la sociedad capitalista. A primera vista acumular es simplemente trasladar al futuro el uso final de recursos ya disponibles para el consumo. Pero sucede que en la sociedad capitalista a ese acto de "renuncia" corresponde una remuneración, la cual solamente se hace efectiva si los recursos acumulados asumen la forma de capital (Furtado, 1983: 18). -Las cursivas son nuestras-.

Con lo cual el progreso técnico no es relevante a menos que asuma una forma específica: la forma de capital, con lo cual Furtado alerta que el objeto relevante de estudio no es el impacto del cambio tecnológico en la producción de la vida material sino el despliegue de relaciones de producción capitalistas en las que la técnica adquiere un sentido determinado.

Pero la lectura de Furtado contiene una dificultad inherente que es idéntica a la señalada por Astarita para el caso de Cardoso y Faletto. La lectura que este autor realiza de Hegel le conduce a una idea del método dialéctico como un método en el cual se jerarquiza la noción de interacción de las partes en la formación 
de un todo con sentido (Furtado, 1965: 29-36). Esta interpretación no logra superar las dificultades de la aproximación analítica, pues desconoce el carácter ontológico del pensamiento dialéctico ${ }^{4} \mathrm{y}$ al mismo tiempo sostiene una relación de exterioridad entre el sujeto y el objeto que, en el sistema de Hegel por ejemplo, está completamente superada.

En este sentido, pueden encontrarse estas ideas en la siguiente síntesis:

Para construir un modelo dinámico, es decir, para explicar un proceso de desarrollo, no bastaría con identificar las interrelaciones entre los múltiples factores que integran un sistema. Siempre será necesario introducir algún elemento exógeno o sea modificar algunos de los parámetros estructurales. Al parecer, existe el consenso de que ese parámetro en permanente modificación en las sociedades modernas es la técnica (Furtado, 1965: 35).

Pero más allá de este diagnóstico, de carácter sobre todo ontológico, la lectura que Furtado logra sobre el proceso de desarrollo de la modernidad capitalista ha sido una de las más profundas que hasta el momento se hayan conocido. La modernización capitalista como fenómeno general y la forma específica que adquiere en los países "subdesarrollados" es, sin duda alguna, un salto cualitativo frente a la visión tradicional de la modelística neoclásica o keynesiana.

En este sentido Furtado entiende que "para comprender las causas de la persistencia histórica del subdesarrollo, es necesario observarlo como parte que es de un todo en movimiento, como expresión de la dinámica del sistema económico mundial generado por el capitalismo industrial" (Furtado, 1983: 35-36).

Dicho esto, Furtado ensaya posibles ideas que expresen la singularidad de los espacios subdesarrollados en el contexto del desenvolvimiento del capital. Pueden extraerse algunos ejemplos de forma sintética de uno de los textos que se han estudiado en este trabajo (Furtado, 1983), destacando sobre todo los puntos problemáticos característicos de los países subdesarrollados:

Marginalidad:

La industrialización retardada $[\ldots]$ se haría en competencia con las importaciones y no con la actividad artesanal preexistente" [...] Las masas demográficas que

4 Sobre esto conviene revisar el nuevo debate sobre el pensamiento dialéctico que intenta fundar una ciencia con una ontología construida desde el sistema hegeliano. Este esfuerzo mantiene intima relación con lo visto en Furtado y el trabajo realizado por ciertos dependentistas pero constituye sin duda una superación dialéctica de éstos (ver por ejemplo, Ollman y Smith, 2008). 
la modificación de las formas de producción priva de sus ocupaciones tradicionales, buscan refugio en sistemas sub-culturales urbanos que sólo esporádicamente se articulan con los mercados, pero que ejercen sobre ellos una fuerte influencia como reservas de mano de obra (Furtado, 1983: 36-37).

\section{Estado:}

Las inversiones infraestructurales y en las industrias básicas, dependen directamente del poder público, o de garantías dadas por éste a grupos extranjeros [...] Pierden así validez muchos de los criterios tradicionalmente usados para diferenciar actividades públicas de actividades privadas (Furtado, 1983: 38).

Clases dominantes, poder e ideología:

La reflexión sobre el desarrollo, al provocar una progresiva aproximación de la teoría de la acumulación a la teoría de la estratificación social y a la teoría del poder (Furtado, 1983: 39).

Estas tres dimensiones, entre otras, deben ser respondidas si se desea teorizar sobre las formas en que el capitalismo se ha desplegado en los países subdesarrollados. Como pudo verse (Furtado: 1965: 65-76), la interpretacion del autor hace pie sobre todo en el complejo sistema de interacciones entre grupos de poder y sus formas ideológicas sobre el progreso social, en el contexto de aún más complejas relaciones exteriores tales que conforman las condiciones para el desarrollo de la ténica, el reparto del excedente y la reproducción del sistema social.

\section{BREVES COMENTARIOS SOBRE EL GIRO NEOCLÁSICO DE COMIENZOS DEL SIGLO XXI: ENTRE EL MERCADO Y LAS INSTITUCIONES}

El derrotero de la teoría del desarrollo y sobre todo de las teorías de la dependencia mantiene una relación evidente con las transformaciones institucionales que se desataron a nivel global y que Jessop resume en su descripción de la formación del Estado Competitivo Schumpeteriano (Jessop, 2008: 169-170) y que devino tras una crisis de lo que el propio Jessop denomina el Estado Nacional de Bienestar Keynesiano (Jessop, 2008: 216-219).

En este contexto la teoría del desarrollo comenzó a redefinirse en los términos del paradigma dominante y fue reescrita sobre la base de la recuperación de los supuestos neoclásicos sobre la sociedad capitalista y su metodología inherente. 
En este sentido, por ejemplo, Fine (2006) afirma que con la llegada del neoliberalismo la vieja economía del desarrollo (es decir, la desarrollada en la segunda mitad del siglo xx) "fue barrida" (was swept), lo que trajo aparejadas ciertas consecuencias poco sorprendentes.

Estas postulaciones teóricas retornan sin escalas a las hipótesis que se analizaban en el caso del modelo Harrod-Domar de crecimiento. Más aún, estos modelos extreman ciertas ideas como la libre movilidad de factores y bienes, la competencia perfecta, la plena ocupación de todos los factores, la estabilidad del equilibrio y por lo tanto la innecesaridad, la inconveniencia y la impotencia del Estado en modificar los resultados "naturalmente" alcanzados. Lo que estos modelos no expresan es, paradójicamente, la naturaleza de la sociedad que estudian; dicha naturaleza queda sumida a una serie de principios antropológicos fuertes (en el sentido que invocan una naturaleza humana poco realista). De hecho, si se recurre a una crítica liberal a estos enfoques, como por ejemplo la realizada por la Escuela Austriaca, se encuentra que su praxiología es sobre todo crítica al hombre maximizador de utilidades y la noción de mercado que la economía neoclásica supone.

Debe destacarse además, que el menú de políticas económicas inspiradas en esta concepción fue su principal expresión institucional, y la crisis social y política despertada en los países subdesarrollados conllevó a una evaluación crítica de dicho "consenso". Esta evaluación crítica dio origen a lo que Fine denomina el pos-Consenso.

La reacción teórica que trajo consigo este pos-Consenso se concentró sobre todo en el irrealismo de los supuestos de la competencia perfecta y del funcionamiento de los mercados y montó una crítica institucionalista sobre los límites del contrato mercantil, manteniendo las suposiciones fundamentales de tradición neoclásica sobre el hombre y el principio primario de socialización: el contrato mercantil.

\section{CONCLUSIONES}

Los distintos enfoques tratados en este trabajo confluyeron de forma más o menos clara en dificultades teóricas comunes: la incapacidad para indagar de manera directa la naturaleza de la moderna sociedad capitalista y su despliegue espaciotemporal.

En general se mostró cómo aquellos elementos fundamentales que constituyen el problema principal de la formación del capitalismo moderno y su reproducción en el tiempo y el espacio quedan ocultos, en la gran mayoría de los 
modelos, a partir de su suposición no explicitada, con lo cual mucho de lo que dichos modelos intentan decir sobre estas suposiciones acaba tomando la forma de una tautología.

Resulta de sumo interés subrayar cómo durante el siglo xx las distintas teorías intentaron superarse unas a otras en el sentido que se remarcó en este trabajo. Rostow intentó poner en movimiento a la manera de una interpretación de la Historia aquello que permanecía estático en Harrod-Domar, quienes a su vez intentaron dinamizar los modelos keynesianos. Germani y Medina Echavarría trataron de superar los límites del determinismo de Rostow profundizando en el esclarecimiento del componente político del fundamento de la modernidad capitalista. Hirschman y Myrdal explicitan un modelo que pone en movimiento las divergencias espaciales, formando así un piso común de análisis para todos los espacios, a pesar de que el propio Hirschman suponía que los "efectos de dispersión y polarización" constituían una ruptura con la economía ortodoxa. A ello aporta la Hipótesis Prebisch-Singer que destaca una condición histórica (la caída secular de los términos de intercambio) como un principio para interpretar la dinámica espacio temporal del desarrollo capitalista tratando se sobreponer el contenido a la forma, pero esta especificidad seguía aún sin problematizar los elementos fundamentales de la sociedad capitalista. Furtado ensaya así una reconstrucción con el explícito objetivo de darle un carácter histórico pero su inclinación por jerarquizar las relaciones de fuerza entre grupos de poder que enarbolan banderas ideológicas sobre el destino de las naciones lo aleja del sentido dialéctico que lleva impresa la sociedad moderna y se aproxima a formas de interpretación sociopolíticas también estáticas.

El propio cambio en el balance de fuerzas a nivel internacional y la instauración de un nuevo modo de acumulación con nuevas formas regulatorias motivó cambios intensos en las perspectivas sobre el desarrollo que desanduvieron la crítica desarrollada en el siglo Xx con la acusación de que constituía un conjunto de recomendaciones morales y políticas disociadas de los enunciados positivos que se corresponden con la ciencia moderna. La nueva teoría del desarrollo basada en la economía neoclásica exacerba la dificultad que se destacó al comienzo: el ocultamiento de suposiciones sustanciales, suposición de los problemas fundamentales que debe resolver y conclusiones tautológicas o invertidas.

En buena medida, las distintas teorías del desarrollo se encuentran expuestas a una crítica intensa, sobre todo porque siendo el desarrollo capitalista su explícito objeto de análisis, muchas veces éste queda disuelto en la modelística y en la forma. Esta ausencia se cubre, en no pocos casos, con recomendaciones normativas y valoraciones políticas que distorsionan aún más las dificultades teóricas. 
El carácter ahistórico de los modelos de desarrollo es casi una paradoja pero al mismo tiempo es una condición de la que no puede librarse si no ensaya transformaciones importantes en sus fundamentaciones sobre todo ontológicas. La teoría del desarrollo debe poner de manifiesto que lo que se está estudiando es el hombre y la sociedad, y la forma en que el hombre y la sociedad traban su existencia en un devenir con sentido. No debe despreciarse el diálogo con la filosofía, desde la que se han desarrollado sistemas muy profundos que intentan penetrar el conocimiento del hombre, la sociedad y su transformación en el tiempo y el espacio. Quizá el más importante de todos ellos y uno de los más polémicos en su interpretación sea el desarrollado por Hegel, a quien Furtado en aquel entonces prestaba especial atención.

\section{BIBLIOGRAFÍA}

Astarita, Rolando, Economía politica de la dependencia y el subdesarrollo. Tipo de cambio y renta agraria en la Argentina, Bernal, Universidad Nacional de Quilmes, 2010.

Cardoso, Fernando Henrique y Enzo Faletto, Dependencia y desarrollo en América Latina. Ensayo de interpretación sociológica, Buenos Aires, Siglo xxI, 2007.

Ferrer, Aldo, La economia argentina. Desde sus origenes hasta principios del siglo XXI, Buenos Aires, Fondo de Cultura Económica, 2008.

Fine, Ben, "The New Development Economics", en Fine, Ben \& Kwame Sundaram Jomo (edit.), The New Development Economics: After the Washington Consensus, London-New York, Zed Books, 2006, pp. 1-20.

Fujita, Masahisa, Paul Krugman y Anthony Venables, The Spatial Economy: Cities, Regions, and International Trade, Cambridge, Massachusetts Institute of Technology, 1999.

Furtado, Celso, Breve introducción al desarrollo: un enfoque interdisciplinario, México, Fondo de Cultura Económica, 1983. - Dialéctica del desarrollo. Diagnóstico de la crisis del Brasil, México, Fondo de Cultura Económica, 1965. , Teoría y política del desarrollo económico, México, Siglo XXI, 1974.

Galindo, Miguel Angel y Graciela Malgesini, Crecimiento económico: principales teorías desde Keynes, Madrid, McGraw Hill, 1994.

Gómez, Ricardo, "El mito de la neutralidad valorativa de la economía neoliberal”, en Energeia, vol. 1, núm. 1, Buenos Aires, UCEs, junio de 2002, pp. 32-51. 
González García, José María, "Las herencias de Kant y de Goethe en el pensamiento de Max Weber", Reis: Revista Española de Investigaciones Sociológicas, núm. 43, Madrid, Centro de Investigaciones Sociológicas, julioseptiembre de 1988 (consultado en abril de 2011), pp. 23-42, disponible en <http://www.reis.cis.es/REISWeb/PDF/REIS_043_04.pdf>.

Gutiérrez, Roberto, "Walt W. Rostow: réquiem por un historiador económico", Ciencia Ergo Sum, vol. 10, núm. 3, Estado de México, UAEMEX, noviembre de 2003-febrero de 2004 (consultado en junio de 2011), pp. 295-303, disponible en <http://redalyc.uaemex.mx/pdf/104/10410307.pdf>.

Hirschman, Albert, "Orto y ocaso de la teoría del desarrollo", en Hirschman, Albert, De la economía a la política y más allá, México, Fondo de Cultura Económica, 1984, pp. 11-39.

Jessop, Robert, El futuro del Estado capitalista, Madrid, Catarata, 2008.

Krugman, Paul \& Masahisa Fukujita, "La nueva geografía económica: pasado, presente y futuro", Estudios Regionales, núm. 004, Alcalá de Henares, Asociación Española de Ciencia Regional, primavera de 2004 (consultado en julio de 2010), pp. 177-206, <http://redalyc.uaemex.mx/ pdf/289/28900409.pdf $>$.

Moncayo Jiménez, Edgard, Evolución de los paradigmas y modelos interpretativos del desarrollo territorial, Santiago de Chile, ILPES-CEPAL-ONu, 2001.

Müller, Alberto, Las teorías del desarrollo: algunas reflexiones desde la actualidad, Buenos Aires, ubA-Ceped, 1996.

Myrdal, Gunnar, Teoría económica y regiones subdesarrolladas, México, Fondo de Cultura Económica, 1959.

Ollman, Bertell \& Tony Smith (edit.), Dialectics for the New Century, Basingstoke, Palgrave Macmillan, 2008.

Roitman, Marcos, Pensamiento sociológico y realidad nacional en América Latina, Chile, exclusiva para Revelion.org, 2005 (consultado en julio de 2011), disponible en <http://www.rebelion.org/docs/619.pdf>.

Rostow, Walt Whitman, Las etapas del crecimiento económico. Un manifiesto no comunista, México, Fondo de Cultura Económica, 1961.

Singer, Hans, "Los modelos keynesianos de desarrollo económico. Un análisis a partir de la obra de Gunnar Myrdal 'Asian Drama”, en Singer, Hans, Estrategia del desarrollo internacional. Ensayos sobre el atraso económico, México, Fondo de Cultura Económica, 1981a, pp. 45-66.

, "Distribución de las ganancias entre los países que invierten y los que reciben préstamos", en Singer, Hans, Estrategia del desarrollo internacional. Ensayos sobre el atraso económico, México, Fondo de Cultura Económica, 1981b, pp. 67-83. 
Weber, Max, "La objetividad del conocimiento propio de las ciencias sociales y de la política social", Revista de Economía Política, Madrid, Centro de Estudios Políticos y Constitucionales, núm. 16, junio-diciembre de 1956 (consultado en julio de 2011), pp. 424-490, disponible en <http://revistas. cepc.es/getData.ashx?MAVqs=-aWQ9MzE0NjkmaWRIPTEwMzcmdXJsPTExJm5hbWU9UkVDUF8wMTZfNDIzLnBkZiZmaWxlPVJFQ1BfMDE2XzQyMy5wZGYmdGFibGE9QXJ0aWN1bG8mY29udGVudD1hcHBsaWNhdGlvbi9wZGY=> 